\title{
The Incidence of Signs and Symptoms of Temporomandibular Disorders Following Third Molar Surgery
}

\author{
Munawar NK ${ }^{1}$, Abd Sattar SS ${ }^{1}$, Hariri F ${ }^{2}$ \\ ${ }^{1}$ Faculty of Dentistry, University of Malaya, Kuala Lumpur, Malaysia \\ ${ }^{2}$ Department of Oro-Maxillofacial Surgical \& Medical Sciences, Faculty of Dentistry, University of Malaya, \\ Kuala Lumpur, Malaysia
}

\begin{abstract}
Third molar surgery, a common dental procedure in Dental Faculty University Malaya, is known to pose many possible complications, one of which is temporomandibular disorder (TMD); a chronic pain concerning the joint apparatus, masticatory musculature and the associated muscle of head and neck region. This research aims (i) to examine the signs and symptoms of TMD following third molar surgery (ii) to study the surgical components of third molar surgery that contribute to the development of TMD (iii) to compare incidence of TMD between operative and non-operative group six months after third molar surgery. A descriptive longitudinal cohort study was conducted by recruiting twenty-two patients as subjects of an operative group, and twenty patients as subjects of a non-operative group. The operative group were examined at baseline, one week, one month, three months and six months after surgery. Each patient underwent a series of Diagnostic Criteria of TMD DC/TMD examination and history questionnaire. In the operative group, we found (i) increased incidence of trismus (92\%), myofascial pain (69\%) and clicking $(77 \%)$ of the patient group at one week review (ii) two new incidences of signs and symptoms of TMD at final review (iii) no significant relationship between operator qualification to development of TMD (iv) no association between degree of impaction to development of TMD (v) no relationship between duration of procedures to development of TMD (vi) there is a significant difference in signs and symptoms of TMD between operative group and control group $(p<0.005)$.
\end{abstract}

Keywords: Facial pain, impacted tooth, oral surgical procedures, third molar, Temporomandibular Joint Disorders

\section{INTRODUCTION}

Surgical removal of third molars is one of the common procedures carried out in the Oral and Maxillofacial Surgery Department here in University of Malaya. On average there are about 28 cases of minor oral surgery (MOS) recorded in the month of May 2015. Surgical procedures, albeit minor or major in nature always come with risks and complications. The mild and common complications of third molar surgery are pain, swelling, trismus and bleeding, which are anticipated and should be mentioned during consent. Besides that, other complications are dry socket $(0.3-26 \%)(1)$, excessive bleeding (0.2$5.8 \%$ ), infection (0.9-4.3\%) (2), injury to adjacent tooth, retained root or foreign body, displaced tooth, periodontal breakdown, fracture of mandible or tuberosity, formation of oro-antral fistula, nerve damage, psychological effect and last but not least, 
temporomandibular disorder $(3,4)$. The anticipated pain, swelling and trismus may be expected and are most severe during the first three days (maximal at 36-48 hours) settling over 5-7 days. At three weeks post-operative, the residual symptoms (if any) should have resolved provided that healing is not complicated by soft or hard tissue infection or inflammation. Sensory functions should have returned to normal as well (4). Thus, the timeline of this study is one week, one month, three months and six months after surgical removal of third molar.

The term temporomandibular disorder (TMD) is defined as a subgroup of craniofacial pain problems that involves the temporomandibular joint (TMJ), masticatory muscles, and associated head and neck musculoskeletal structures. According to a study done among the adults in United States, $40 \%-75 \%$ of the population develop signs of TMD, and $33 \%$ of the population having at least one symptom of TMD (5). In Toronto, Canada a study on 1002 individuals had reported that $48.8 \%$ of the population is associated with symptoms of temporomandibular joint disorders (6). Patients with TMD most frequently present with pain, limited or asymmetric mandibular motion and TMJ sounds. Other common associated symptoms are ear pain and stiffness, tinnitus, dizziness, neck pain and headache. Physical assessment consists of observation and measurement of mandibular motion (maximum inter-incisal opening, lateral movements and protrusions), palpation of the muscles of mastication (masseter, temporalis, medial and lateral pterygoid muscles), cervical musculature at the posterior and anterior neck, palpation or auscultation of TMJ and examination of the oral cavity, dentition, occlusion and salivary glands.

The causes of TMD are complex and multifactorial. There are three types of factors that may lead to development of TMD: (i) predisposing factors; factors that increase the risk of TMD (lii) initiating factors; factors causing the onset of TMD (iii) perpetuating factors; factors that interfere with healing or enhance progression of TMD (7). Example of initiating factor is trauma or adverse loading to TMJ, while perpetuating factors may include behavioral, social, emotional and cognitive factors. Based on a retrospective study carried out in a TMD clinic in France, the two most prevalent factors were toothclenching ( $77 \%$ of patients) and psychological stress $(59.3 \%)$, followed by antecedents of wisdom tooth extraction $(34.3 \%)(8)$. In line with the understanding that third molar removal is a risk factor for TMD, Deangelis has highlighted the importance of preoperative evaluation of the TMJ apparatus prior to carrying out the surgery $(9,10)$.

The development of signs and symptoms of TMD following third molar removal is a logical possibility since the surgery involves opening the mouth wide for a prolonged period and the force applied to remove the impacted third molar might prove to be detrimental to the nearby anatomical structure; i.e. the temporomandibular joint. The location and severity of third molar impaction could also be a contributing factor considering the proximity of third molar to the TMJ.

The clinical protocol used in this research, the Diagnostic Criteria for Temporomandibular Disorders (DC/TMD) is an established diagnostic instrument for TMD which was first published in 1992. It has been revised and modified since to provide clinicians with the complete and concise method of examination, diagnosis and classification of the various subtypes of TMD. The advantages of DC/TMD are: i) guidelines for systematic, clinical examination of TMD, ii) measurable terms for major clinical variables, iii) demonstrated reliability and iv) combination of recorded physical findings involving behavioral, psychological and psychosocial status (11).

Although third molar surgery is carried out frequently here in Oral and Maxillofacial Surgery department of University of Malaya, there had been no investigation done to study the incidence of Temporomandibular Disorder following the surgery. Therefore, it is of paramount importance for more studies to be done to investigate the association of TMD with third molar surgery.

Hence, this study is conducted with the following aims: (i) to examine the presentation of signs and symptoms of TMD after third molar surgery, (ii) to examine the various aspects of third molar surgery that could lead to development of TMD, and (iii) to compare the incidence of TMD between patients and a non-operative control group six months after third molar surgery.

\section{MATERIALS AND METHODS}

Study Design:

This descriptive longitudinal (cohort) study is done under the authorization of University of Malaya Dental Faculty Ethics Committee. The study was conducted at the Oral and Maxillofacial Surgery Department, Dental Faculty University Malaya, Kuala Lumpur, Malaysia. The subjects taken for this study were all recruited from the clinic's out-patient list. Subjects were briefed on the study objectives and procedures before signing the informed consent. The study is done within an eight-month time-frame; the first two months were utilized to recruit patients followed by a six-month evaluation period. 


\section{Study Population:}

This study was a prospective cohort study implemented by having two study groups of subjects. The first group (test group) consists of twentytwo subjects $(n=22)$ presenting for evaluation and extraction of third molar. The second group (control group) is the non-operative group comprising of twenty patients. The test group comprised of any patient indicated for third molar surgery presenting with none of the following exclusion criteria: patients with history of mandibular third molar surgery, patients presenting with TMD or painful physical conditions, bruxism, psychiatric or medical conditions that might impair communication or compliance, contraindications to analgesics, use of analgesics or anti-inflammatory drugs within 12 hours preceding assessment, concomitant use of sedatives or microsomal enzyme inducers. In the first two months of study, patients scheduled for third molar surgery with no exclusion criteria were enlisted under the test group. Evaluation and examination was done on each subject at baseline (pre-operation), one week, one month, three months and six months after third molar surgery.

The second group (control group) was extracted from the population with no indication of third molar surgery. The subjects of this group have the following inclusion criteria: aged at least 18 years old, both genders, no indication for third molar surgery treatment before or during the study period. The control group were excluded from any history of mandibular third molar surgery, other painful physical conditions, psychiatric or medical conditions that might impair communication or compliance with the study procedures, use of NSAIDS or other analgesic drugs within 12 hour preceding the assessment and concomitant use of sedatives.

\section{Surgery:}

The third molar surgeries for the test group were carried out in accordance to several standardized parameters. In terms of the surgical techniques, the following procedures were made constant: local anaesthesia (2\% lidocaine) was administered for inferior alveolar nerve (ID) block, buccal infiltration and lingual infiltration with no specific dosage. This is followed by an incision and opening of mucoperiosteal flap, bone removal with round bur, tooth extraction, socket debridement and wound closure with Vicryl 3-0 or 4-0. On the other hand, the following parameters were used as variables for this study; a) Qualification level of operator: Undergraduate/ Postgraduate/ Lecturers (b) degree of impaction of mandibular third molar (according to Pell and Gregory Classification, as described in Fig. 1 and
Table 1), (c) Duration of the procedure (d) usage of mouth prop (12). Post operatively, the patients were prescribed with analgesic and antibiotic (Amoxycillin and Paracetamol) according to individual needs. This was followed by patient evaluation one week, one month, three months and six months after surgery.

In the control group, assessment and evaluation were made at baseline and six months after the initial examination. Within the six months, any emerging signs and symptoms experienced by the subjects were reported and recorded.

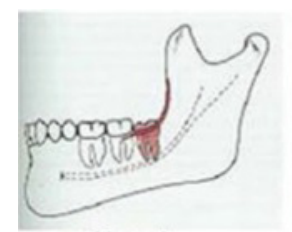

Class I

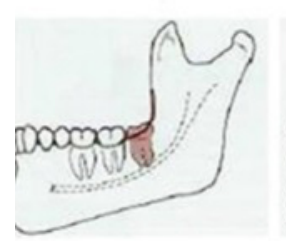

Class A

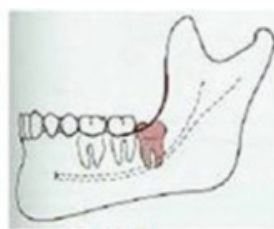

Class II

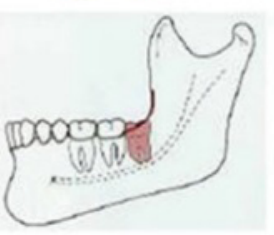

Class B

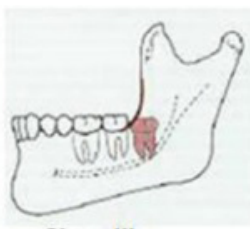

Class III
Figure 1: Pell and Gregory's Classification

Table 1: Pell and Gregory's Classification

\begin{tabular}{ll}
\hline Type & Description \\
\hline Class I & Mesiodistal diameter of crown of $3^{\text {rd }}$ \\
& molar is completely anterior to the \\
& anterior border of mandibular ramus.
\end{tabular}

Class II One half of crown of $3^{\text {rd }}$ molar is in the ramus of mandible.

Class III All or more than half of the crown of $3^{\text {rd }}$ molar is in the ramus of mandible.

Position A The highest portion of the impacted tooth is on a level same or above the occlusal plane of $2^{\text {nd }}$ molar.

Position B The highest portion of the impacted tooth is between the occlusal plane and cervical line of $2^{\text {nd }}$ molar.

Position C The highest portion of the impacted tooth is below the cervical line of $2^{\text {nd }}$ molar.

\section{DC/TMD Method}

The subjects were examined according to the Diagnostic Criteria for TMD (DC/TMD) which is a validated and reliable evidence-based instrument used to identify patients presenting with TMD signs 
and symptoms $(13,14)$. This protocol comprises of clinical examination (Axis I) and history questionnaire (Axis II). Axis I includes demographics, symptom questionnaire and clinical examination of the mouth opening, the presence of pain upon palpation of the masticatory muscles and the TMJ, as well as the presence of any noises of the joint (e.g.: clicking, crepitus) during function. Two examiners were involved in the process and they were calibrated based on the comprehensive guidelines given in the DC/TMD instrument. Axis II on the other hand measures jaw disability, parafunctional habits, psychologic status and psychosocial functioning of subjects.

\section{Statistical Analysis}

In order to analyze the data collected and achieve the objectives, Fisher's Exact test was used to determine the association between the variables. It was used to determine whether the surgical components have any bearings on TMD diagnoses post-operatively (second objective) and to test for differences between the test and control group in terms of TMD diagnoses after 6 months (third objective). The choice of this statistical test is due to the categorical nature of the variables and the small sample size. These statistical calculations were carried out using the Statistical Package for the Social Sciences (SPSS) version 12.0.1.

\section{RESULTS}

A total of 22 patients were selected as patients for the operative group ( 8 men, 14 women) with the mean age of 24 years. Twenty subjects ( 6 men, 14 women) were recruited for our control group with the mean age of 23 years. A majority of the subjects are Malay and Chinese with an approximately equal distribution of $55 \%$ and $36 \%$ respectively, with the exception of two people from the operative group being Indians. More than half of the subjects are not married with educational background of at least secondary school. Their monthly household incomes fall into the categories of RM 1000 - RM 3000 (54\%), RM 3000 - RM 5000 (32\%) and above RM 5000 (14\%).

All patients from the operative group who came to the department were indicated for surgical removal of impacted mandibular third molar, out of which 9 $\%(n=2)$ of them also had forceps extraction of the opposing maxillary third molar. The surgeries were carried out by operator with varying qualifications; either by undergraduate dental students, oral surgery trainees or dental officers at the Faculty of Dentistry, University of Malaya. The Pell and Gregory Classification were used to classify the degree of impaction and nearly $41 \%$ of the patients presented with class $1 \mathrm{~A}$. The surgical procedure for the operative group patients were mostly completed in less than an hour and only 7 of them lasted for about 1-2 hours. Mouth prop was used in all the surgeries.

\section{Axis I: Solitary symptoms and signs of TMD in} patients during the 6-month observation period

Examination on the signs and symptoms of TMD was performed based on the DC/TMD Examination Form, in which assessment of facial pain and headache location, opening patterns, jaw movement limitations, TMJ noises, joint locking and muscle/ TMJ pain were done. It was discovered, from Figure 1 , that $32 \%$ of patients $(n=7)$ have reduced non painful jaw opening one week after surgery from an average of $45 \mathrm{~mm}$ (pre-operative) to $37 \mathrm{~mm}$ (postoperative). At final review, $46 \%$ of the patient group $(n=19)$ reverted to their baseline mouth opening values with an average of $46 \mathrm{~mm}$ with the exception of three patients who presented with reduction of $5 \mathrm{~mm}$ from baseline and average mouth opening of $45 \mathrm{~mm}$. Facial pain and headache were recorded as common findings in all patients in this group during the one week review which resolved in the following one month, three months and six months reviews. The incidence of clicking of TMJ is recorded in 9 patients at baseline and three of the 13 patients who came for one week review were still having clicking TMJs. There was no new incidence of clicking at one week and final review among the rest of the patients. Muscle pain upon palpation was recorded in one patient at baseline with 3 new incidences at one week review. The newly developed muscle pain was mainly found in the masseter muscle (insertion). Only one patient reported persistent muscle pain at all post-operative reviews. This patient also presented with newly developed TMJ pain at 1-month, 3-month and 6-month reviews. Additionally, at 3-month review, there was also another new incidence of supplemental muscle pain upon palpation, at the posterior mandibular region. These two patients, with newly developed TMD, were found to have no significant surgical components that could have contribute to their TMD manifestation. Both of these patients had degree of impaction $1 \mathrm{~A}$, duration of procedure was less than an hour, and the surgeries were carried out by lecturers.

\section{Axis II: History Questionnaire}

It is well accepted that psychological factors and physical functioning are closely associated to the development of TMD. These psychological and 
physical aspects have been comprehensively accounted for under Axis II of DC/TMD instrument under several domains: (1) pain intensity, (2) nonspecific physical symptoms and functioning and (3) emotional functioning. At baseline, most of the patients were reported as having mild physical symptoms, mild depression and anxiety scores. Only two patients presented with Graded Chronic Pain Scale score of Grade II-High, indicating painrelated interference in daily lives. Three patients complained of difficulty in chewing. At the 1 week review appointment, one patient presented with newly-developed symptoms of psychological distress while another patient recorded an increase in the depression score. Limitation of jaw activities is anticipated following third molar surgery. Six out of 13 patients who came for 1 week review complained of difficulty in chewing tough food, making facial expressions, talking and yawning. Nevertheless, the jaw activities of all the patients returned to normal ranges on the subsequent reviews. Parafunctional habits such as clenching and grinding has been found to have significant relationship with symptoms of myofascial pain. The incidence of such habits at baseline and reviews were found to be $19 \%$ in the patient group $(n=4)$, with 3 patients having bruxism and one patient having clenching habit during the day. Meanwhile for the control group at baseline and 6 -month review, only $15 \%$ of the subjects $(n=3)$ exhibited parafunctional habit.

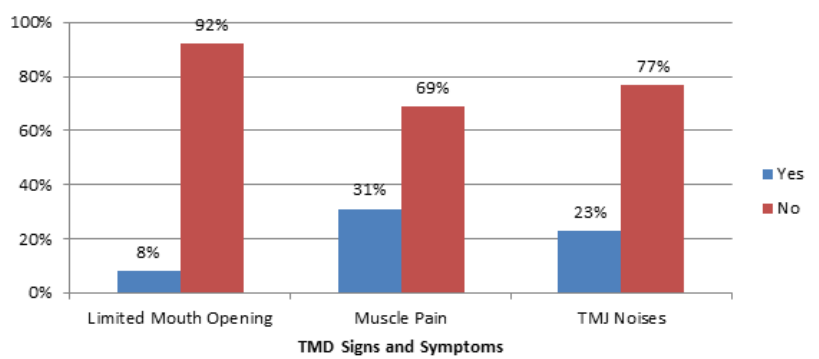

Figure 2: Incidence of TMD signs and symptoms at 1-week review in the test group $(n=13)$

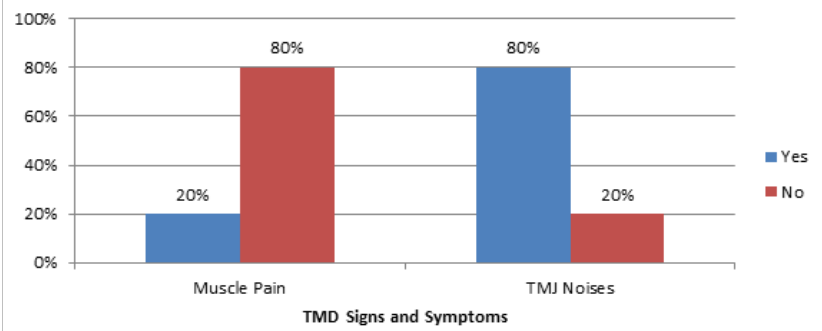

Figure 3: The incidence of TMD signs and symptoms in the test group at 3-month review

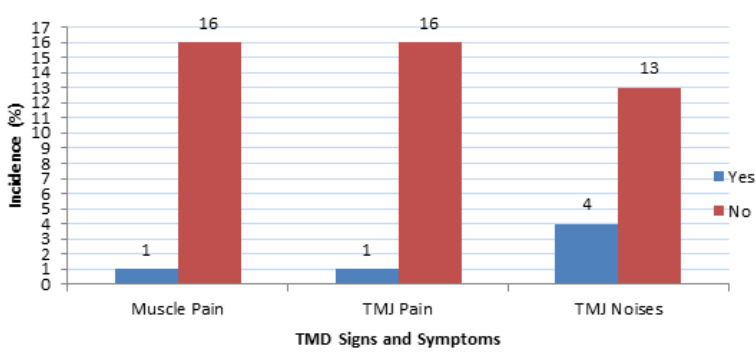

Figure 4: The incidence of TMD signs and symptoms in the test group at 6-month review

Table 2: The newly developed TMD signs and symptoms at 6-month review

\begin{tabular}{lll}
\hline TMD & No. of Patients (\%) & No. of Control (\%) \\
\hline $\begin{array}{lll}\text { Baseline } \\
\text { Yes }\end{array}$ & $9(41)$ & $2(11)$ \\
No & $13(59)$ & $18(89)$ \\
Final Review & & \\
Yes & $11(50)$ & $2(11)$ \\
No & $11(50)$ & $18(89)$ \\
\hline
\end{tabular}

Table 3: The effect of varying surgical components to the development of TMD at final review

\begin{tabular}{|c|c|c|}
\hline \multirow[t]{2}{*}{ Surgical Components } & \multicolumn{2}{|c|}{$\begin{array}{l}\text { Development of TMD } \\
\text { Signs and Symptoms }\end{array}$} \\
\hline & Yes & No \\
\hline \multirow[t]{2}{*}{$\begin{array}{l}\text { Operator Qualification } \\
\text { Undergraduate } \\
\text { Postgraduate/Lecturer }\end{array}$} & 4 & 5 \\
\hline & 5 & 8 \\
\hline \multirow{3}{*}{$\begin{array}{l}\text { Duration } \\
\text { Less than } 1 \text { hour } \\
1-2 \text { hours }\end{array}$} & & \\
\hline & 7 & 11 \\
\hline & 2 & 2 \\
\hline \multirow[t]{2}{*}{$\begin{array}{l}\text { Degree of Impaction } \\
\text { Less than half of crown in ramus } \\
\text { More than half of crown in ramus }\end{array}$} & 8 & 10 \\
\hline & 1 & 3 \\
\hline
\end{tabular}

\section{DISCUSSION}

This study is focused on the investigation of the development of signs and symptoms of temporomandibular disorder following third molar surgery between two groups of subjects (patient group and control group) within a 6-months period. At baseline, $9(41 \%)$ patients from the test group and 2 $(11 \%)$ patients from the control group were examined to have existing solitary signs and symptoms of TMD (Table 1). Final examination was carried out at 6 months and 3 months post-surgical extraction. 
Among the patients that were examined at final review, it was found that two patients from test group $(9 \%)$ reported incidence of newly developed signs and symptoms of TMD. There were no significant changes among the remaining healthy patients and patients with existing signs and symptoms of TMD. However, at one week post-surgical extraction, we found an increase occurrence of signs and symptoms of TMD among the subjects of the operative group. The symptoms were limited mouth opening, TMJ noises on palpation and muscle/ TMJ pain. Subjects were also found to have increased pain-related physical disability. Pain, swelling and trismus were the commonest complaints following third molar surgery (11) and they are thought to arise from inflammatory response which is a direct and immediate consequence of the surgical procedure (15).

Upon clinical assessment of the test group at 1 week review as shown in Figure 1, there was increased incidence of trismus (92\%), myofascial pain $(69 \%)$ and clicking $(77 \%)$ as compared to baseline findings. At the 3-month and 6-month reviews, as in Figure 2, it was reported that there were only two new incidences of newly developed TMD. The signs and symptoms reported were myalgia of the temporalis muscle and posterior digastric muscle as well as pain around the lateral pole of TMJ.

One of the aims of our study is to investigate the association between third molar surgery and the development of TMD. The surgical parameters that were taken into account were operator qualification, degree of impaction and duration of procedure. However, it was found that none of the said parameters have any significant adverse effect on the temporomandibular joint. Our statistical analysis using Fisher's exact test yielded insignificant results correlating degree of impaction, operator's qualification and duration of procedure towards TMD with $p=0.616, p=1.00, p=1.00$ respectively. The two patients with positive newly developed TMD were both operated by lecturers in less than an hour with the same degree of impaction; involving less than half of crown in the ramus. Alongside with these findings, the rest of the operative patient group who did not develop TMD after third molar surgery also have various operator qualification, duration of procedure and degree of impaction. To reinstate, based on our results, there is no relationship between the surgical components and the development of TMD. On the contrary, some literatures have reported otherwise. These literatures have stated that increase in the force used, duration of time and degree of impaction of the third molar would increase the post-operative complication, one of which is TMD (16). Third molar removal involves prolonged maximum opening with exertion of high magnitude of force during elevation of the tooth. Such uncomfortable nature of procedure could strain the ligaments and muscles of the TMJ with possible disc displacement (18).

Third molar surgery, involving deeply impacted tooth with close proximity to the mandibular canal pose a risk of traumatizing the nerve in hard and soft tissue, leading to central and peripheral neurons sensitization and subsequently increased pain perception (19). Apart from that, the inflammation at surgical site could contribute to sensitization which could spread to adjacent structures leading to chronic pain of the masticatory apparatus (19). As mentioned earlier, there were two patients who presented with newly developed signs and symptoms of TMD at the 3-months and 6-months reviews. The signs and symptoms recorded were persistence of muscle pain (posterior digastric muscle, temporalis muscle and medial pterygoid muscle) and pain around lateral pole of TMJ. This occurrence could be attributed to the peripheral or central sensitization after the surgery. The said sensitization could tip the balance of pain threshold towards neuropathic pain, theoretically emitting positive signs and symptoms of TMD (20). Therefore, from our findings, the signs and symptoms experienced by the two patients from the test group resemble that of myofascial TMD and arthralgia.

Out of the 20 subjects that were recruited in the control group, Fisher's Exact Test showed significant statistical difference; $p=0.017$ between test group and control group at baseline. As shown in Table 1, it was found that $11 \%(n=2)$ of them had preexisting signs and symptoms of TMD. This finding was consistent at the final 6 months review, suggesting that there was no new incidence of TMD among the control group. Comparing the incidence of signs and symptoms of TMD between the patient group and control group, at baseline, $41 \%$ of the patients from operative group had preexisting TMD. However, at final review, our finding revealed that there is an increase in the number of patients $(50 \%)$ with TMD. As a result, our study indicates that there is an increment of $9 \%(n=2)$ in the incidence of TMD following third molar surgery in the patient group. Fisher's exact test showed a positive significant difference; $p=0.007$ between the patient group and control group at 6-month review.

Apart from clinical examination, the DC/ TMD incorporates assessment of jaw disability during function, non-specific physical symptoms, psychological status and oral behavior of the subjects. This is in line with the current evidence supporting the detrimental effect of these aspects on signs and symptoms of TMD $(24,25,26)$. One of which is a study done is Japan, with positive relationship between emotional stress and TMD (27). At baseline, 
patients with existing signs and symptoms of TMD also demonstrated relatively high psychological distress, disability score and unfavorable oral habits. At the final 6 months review, these patients have consistent report of such manifestations. Hence these psychological and oral behavioral components can be said to be characteristic findings among patients with TMD. Most of the patients have depression scores of mild to moderate levels, and were reported as having some form of parafunctional habits such as clenching and grinding during sleep.

Myofascial pain was reported in subjects with clenching, facial trauma, depression, somatization and talking with the phone resting on the shoulder. According to the study by Bonjardim LR et al., and Mazzetto MO, it was found that TMD was aggravated by heightened level of anxiety $(21,22$, 23). Magnitude of pain threshold was found to be reduced in patients with depression, stress and mood and concurrently increase risk of TMD. Hence, pain sensitivity associated with psychological factors was found to influence risk of TMD. The common somatic and psychological complains that the patients with TMD normally present with are fatigue, headache and trouble sleeping. These physical symptoms are commonly associated with TMD (24). The relationship between insomnia and non-specific pathogenic pain in the TMJ area suggest a central sensitivity to pain which could have an etiologic role in idiopathic pain disorder (25).

Many literatures had come to an agreement that parafunctional habits have an influence on the development of $\operatorname{TMD}(26,27)$. At the final review, from the patient group, $45 \%$ of the patients with TMD and from the control group, all subjects with TMD were discovered to exhibit parafunctional habits of different frequencies. Parafunctional habits play an etiological role in TMD due to the excessive force exerted on the dentition and masticatory structures through their prolonged use when carrying out these habits (26). From our study, the patients shared some common oral behaviors, such as clenching, grinding, chewing on one side and placing pressure on jaw when sleeping. From previous studies, more emphasis had been given on the effects of clenching and grinding on TMD. Clenching habits cause masticatory muscle pain through trauma to muscle fibers or decreased blood supply (26). Apart from that, clenching and grinding could contribute to TMJ disc displacement. This is due to abnormal distribution of stress in the disc during the static and dynamic movements of the joint structures. In addition, unilateral chewing habits could lead to remodeling of the TMJ apparatus (28). It was discovered that the favorable chewing side exhibit a higher condylar path and flatter lateral anterior guidance angles (28).
Postural habits involving prolonged applied pressure to the jaw such as abnormal sleeping positions and jaw cupping habit are related to abnormal head, neck and jaw posture (28). Abnormal posture could alter the neuromuscular influences on masticatory structures hence, inducing incidence of TMD signs and symptoms (28).

These psychological and behavioral aspects of TMD assessment covered under Axis II of $D C$ / TMD is of paramount importance in the treatment planning stage of this chronic pain condition. TMD is a multifaceted disease with various clinical presentations and etiological factors. Hence, it requires a multidisciplinary approach comprising of clinical/surgical, psychological and behavioral managements.

There were limitations to our study. Our tight clinical schedule overlaps with the minor oral surgery schedule, as most of the surgeries were done during weekdays in the morning. Moreover, the semester break was occupied with postgraduate examinations and conference, so there were less scheduled surgeries. Finally, we experience time constrain when doing this study. This is because of the limited time provided and the nature of our study that requires 6 months follow up. Hence we had a narrow time window to recruit subjects for our study. All these limitations resulted in our low sample size thus, resulting in the use of non-parametric test of a weaker statistical power. Therefore, further studies of a larger sample size on the incidence of signs and symptoms of TMD are recommended in the future in order to generate more statistically significant results.

\section{CONCLUSION}

This prospective cohort study found that there are new development of solitary signs and symptoms of TMD following third molar surgery in the operative patient group at final reviews of 3 months and 6 months. There was increased incidence of TMD signs and symptoms at 1 week review which mainly consists of limited mouth opening, muscle pain and TMJ noises with complete remission to baseline, except for one patient with persisting presentation. Additionally, there was one new incidence of myofascial pain related to TMD at 3 months final review. Since there was no change in the number of positive TMD in our control group at 6 months, within the limitation of this study, it can be concluded that there is statistically important difference of newly developed TMD between patient and control group after 6 months. Finally, among the patient group, there is no significant association between the surgical components and the development of signs and symptoms of TMD at final reviews. 


\section{Acknowledgements}

First and foremost, we would like to express our heartfelt gratitude to Miss Najihah Lokman for the statistical consultation provided throughout this study. Likewise, the authors greatly thank the dental officers and staffs of the Oral and Maxillofacial surgery Department, University of Malaya for their assistance in conducting this research.

\section{Declaration Of Interest}

The authors report no conflicts of interest. The authors alone are responsible for the content and writing of the paper.

\section{References}

1. Contar CMM, de Oliveira P, Kanegusuku K, Berticelli RS, Azevedo-Alanis LR, Machado MA. Complications in third molar removal: a retrospective study of 588 patients. Med Oral Patol Oral Cir Bucal. 2010; 15(1): e74-8.

2. Bui $\mathrm{CH}$, Seldin EB, Dodson TB. Types, frequencies, and risk factors for complications after third molar extraction. J Oral Maxillofac Surg. 2003; (12): 1379-89.

3. Dimitroulis, G. A synopsis of minor oral surgey. Woburn: Butterworth-Heinemann. 1997: pp 159-69.

4. Shugars DA, Benson K, White RP, Simpson KN, Maynor G, Bader JD. Developing a measure of patient perceptions of short-term outcomes of third molar surgery. Abstracts of presentation at AAOMS Scientific Meeting 1995.

5. Scrivani SJ, Keith DA, Kaban LB. Temporomandibular disorders. N Engl J Med. 2008; 2693-2705.

6. Locker D, Slade G. Prevalence of symptoms associated with temporomandibular disorders in a Canadian population. Community Dent Oral Epidemiol. 1988; 16: 310-313.

7. Sharma S, Gupta DS, Pal US, Jurel SK. Etiological factors of Temporomandibular Joint Disorders. Natl J Maxillofac Surg. 2011; 2 (2): 116-19.

8. Robin $\mathrm{O}$, Chiomento A. Prevalence of risk factors for temporomandibular disorders: a retrospective survey from 300 consecutive patients seeking care for TMD in a French dental school. Int J Stomatol Occlusion Med. 2010; 3(4): 179-86.

9. DeAngelis AF, Chambers IG, Hall GM. Temporomandibular joint disorders in patients referred for third molar extraction. Aust Dent $\mathrm{J}$. 2009; 54(4): 323-325.

10. Deliverska EG, Petkova M. Complications after extraction of impacted third molars- literature review. J of IMAB. 2016; 22(3): 1202-1211.

11. Oginni FO, Ugboko VI, Assam E, Ogunbodede EO. Postoperative complaints following impacted mandibular third molar surgery in lleIfe, Nigeria. South Afr Dent J. 2002; 57(7): 2648.

12. Lima CJ, Silva LC, Melo MR, Santos JA, Santos TS. Evaluation of the agreement by examiners according to classifications of third molars. Med Oral Patol Oral Cir Bucal. 2012; 17(2): e281e286.

13. Ohrbach R, Gonzalez $\mathrm{Y}$, List $\mathrm{T}$, Michelotti A, Schiffman E. Diagnostic criteria for Temporomandibular Disorders (DC/TMD) clinical examination protocol: Version 02June2013. www.rdc-tmdinternational.org. Accessed on $2^{\text {nd }}$ May 2015.

14. Schiffman et al. Diagnostic criteria for Temporomandibular Disorders (DC/TMD) for clinical and research applications: recommendations of the international DC/TMD consortium network and orofacial pain special interest group. J Oral Facial Pain Headche. 2014; 28 (1): 6-27.

15. McGrath C, Comfort MB, Lo EC, Luo Y. Changes in quality of life following third molar surgerythe immediate postoperative period. $\mathrm{Br}$ Dent $\mathrm{J}$. 2003; 194(5): 265-268.

16. Kasapoğlu C, Brkić A, Gürkan-Köseoğlu $B$, and Koçak-Berberoğlu H. Complications following surgery of impacted teeth and their management, a textbook of advanced oral and maxillofacial surgery. InTech; 2013. doi: $10.5772 / 53400$

17. Macfarlane TV, Blinkhorn AS, Stevenson LJ, Coulthard P. Third molar removal and orofacial pain: a population-based survey. J Oral Maxillofac Res. 2010; 1(3): e4.

18. DeAngelis AF, Chambers IG, Hall GM. Temporomandibular joint disorders in patients referred for third molar extraction. Aust Dent J. 2009; doi: 10.1111/j.18347819.2009.01157.x.

19. GI Juhl, Jensen TS, Norholt SE, Svensson $P$. Incidence of symptoms and signs of TMD following third molar surgery, a controlled, prospective study. J Oral Rehabil. 2009; 36: 199-209.

20. Berge TI. Incidence of chronic neuropathic pain subsequent to surgical removal of impacted 
third molars. Acta Odonto Scand. 2002; 60(2): 108-12.

21. Bonjardim LR, Gavião MB, Pereira LJ, Castelo PM. Anxiety and depression in adolescents and their relationship with signs and symptoms of temporomandibular disorders. Int J Prosthodont. 2005; 18(4): 347-52.

22. Mazzetto MO. Alteranues psicossociais em sujeitos com desordens crßnio craniomandibulares. J Bras. Oclusyo, ATM \& Dor Orofacial 2001; 1: 223-43.

23. Syed RA, SyedaAA, Katti G, Arora V. Prevalence of temporomandibular joint disorders in outpatients at Al-Badar Dental College and Hospital and its relationship to age, gender, occlusion and psychological factors. J Indian Acad Oral Med Radiol. 2012; 24(4): 261-8.

24. Aldiéris AP, Zuim PRJ, Monteiro DR, Ribeiro Pdo P, Garcia AR. Relationship between psychological factors and symptoms of TMD in university undergraduate students. Acta Odontol. Latinoam. 2010; 23(3): 182-7.

25. Buljan D. Psychological and psychiatric factors of temporomandibular disorders. Medical Sciences. 2010; 34: 119-33.
26. Michelotti A, Cioffi I, Festa P, Scala G, Farella M. Oral parafunctions as risk factors for diagnostic TMD subgroups. J Oral Rehabil. 2010; 37: 157-62.

27. H. Ohmi, M. Kato, M. Meadows. Relationship between type a behaviour patterns and risk of temporomandibular disorders in Japanese students. J Rural Med. 2016; 11(2): 77-80.

28. Santana-Mora U, López-Cedrún J, Mora MJ, OteroXL,Santana-PenínU.Temporomandibular disorders: the habitual chewing side syndrome. PLoS One. 2013; 8(4): e59980.

\section{Corresponding author:}

Dr Firdaus bin Hariri

Department of Oro-Maxillofacial Surgical \& Medical

Sciences

Faculty of Dentistry

University of Malaya, 50603,

Kuala Lumpur,

Malaysia.

Email: firdaushariri@um.edu.my

Tel: +603-79674807 